\title{
Perception des risques nucléaires
}

\author{
J. BRENOT*, S. BONNEFOUS*, Ph. HUBBERT*
}

(Manuscrit reçu le 6 novembre 1995,
révisé le ler juillet 1996, accepté le 3 septembre 1996)

RÉSUMÉ La perception d'un risque par l'individu dépend de nombreux facteurs, certains propres à l'individu (psychologie, connaissance, expérience), d'autres descriptifs du contexte sociétal (culture, idéologie), d'autres enfin relatifs à la nature du risque. Le mot risque possède des sens divers et il est appréhendé de façon différente par les trois catégories d'intervenants impliqués dans la maîtrise du risque, à savoir les techniciens, les gestionnaires et les individus du public. Plusieurs modèles pour décrire comment s'élabore la perception sont présentés. Les dimensions intervenant dans la perception sont énumérées et une classification des divers risques est proposée. A partir d'enquêtes faites au sein du public, la perception du risque nucléaire est située par rapport à celle d'autres risques. Enfin, des résultats sont donnés concernant la confiance dans l'information diffusée et la crédibilité des organismes et institutions.

ABSTRACT Many factors are involved in the perception of a risk by individuals. Some are individual related (psychology, knowledge, experience), others describe the social environment (culture, ideology), others at end precise the nature of the risk. The word risk has various meanings and is understood differently by the three main categories involved in risk management, that is engineers, administrators and lay public. Several models which describe the perception phenomenon are presented. The dimensions affecting perception are listed. Based on public opinion poll data, a risk taxonomy is proposed, perceived nuclear risks are compared with other risks, and results are given concerning trust in the information diffused and the credibility of those in charge of nuclear activities.

\section{Introduction}

La prise en compte dans la gestion des risques technologiques de leur perception par le public constitue un fait relativement récent. C'est au début des années 1970 que la recherche du "risque nul" a été jugée irréaliste, notamment dans le cas des cancérogènes pour lesquels il n'était pas possible a priori de postuler un niveau d'innocuité. Les principes réglementaires ont alors été fondés sur la recherche de pratiques et de niveaux de risque "acceptables" (Travis et Hattemer-Frey, 1988). Dans le domaine nucléaire, la Commission internationale de protection radiologique (CIPR) a développé dès ces années un système de limitation des expositions aux rayonnements pour diminuer les

Institut de Protection et de Sûreté Nucléaire, Département de Protection de la Santé de l'Homme et de Dosimétrie, Service d'Évaluation et de Gestion des Risques, IPSN, BP 6, 92265 Fontenay-aux-Roses Cedex, France. 
probabilités d'effets radio-induits. L'objectif assigné était que toute activité devait s'exercer dans des conditions de protection telles que les expositions aussi bien des travailleurs que du public soient maintenues aussi bas que raisonnablement possible compte-tenu des facteurs économiques et sociaux. Ce principe connu sous le nom de "ALARA" ("As Low As Reasonably Achievable", ICRP Publ. 26, 1977) est maintenant l'un des trois principes de base de la gestion des risques radiologiques.

Cependant, la gestion d'une situation ou d'une activité en risque acceptable n'a pas suscité l'adhésion des populations espérée. Le risque n'a pas le même sens pour tous et la définition de l'acceptable est très dépendante du contexte de la situation considérée. Des divergences d'opinion entre gestionnaires et public, de multiples contestations se sont manifestées, notamment à propos des programmes énergétiques. Les difficultés de mise en place des programmes ont conduit les décideurs des pays concernés à lancér des études sur le pourquoi des réticences et des refus des populations. L'idée première a été d'introduire le terme "risque perçu" et d'imputer les réactions négatives observées à une méconnaissance du "risque objectif". Cependant le manque d'information n'a pas suffi pour expliquer les attitudes des populations face au risque et d'autres raisons ont été recherchées.

Toutes les études sur la perception des risques répondent à l'un des deux objectifs suivants. Le premier objectif est de connaitre comment le public évalue les risques et de comprendre le pourquoi des différences observées dans les divers groupes sociaux. Le second objectif est de relier les perceptions aux attitudes et comportements. Il s'agit là de gestion. Quatre volets sont particulièrement concernés : l'information sur le risque, la communication entre les divers intervenants du système de maîtrise du risque, l'établissement de modes de gestion acceptables et l'acceptabilité des actions de sécurité.

Les travaux sur les risques perçus ont été initiés par Slovic et al. aux EtatsUnis (1980). Ils se poursuivent toujours, et ceci s'explique par la multiplicité des situations à risques sujettes à contestation, la variété des groupes d'individus concernés, la diversité des contextes sociaux. En France, l'IPSN conduit depuis 1977 des études relatives à la perception des risques, auxquelles participe la Commission des Communautés européennes. Les travaux menés sont de type conceptuel (notions de risque, d'acceptabilité) ou bien s'appuient sur des enquêtes d'opinion. La plupart des résultats chiffrés mentionnés ci-après sont issus de ces enquêtes mais ils sont de portée plus générale car très semblables à ceux obtenus dans de nombreux pays d'Europe et en Amérique du Nord.

\section{La maîtrise du risque : trois acteurs en présence}

La première difficulté rencontrée lorsqu'il s'agit d'analyser le phénomène auquel renvoie le risque perçu tient au fait qu'il n'existe pas une définition 
unique du mot risque. Son sens va dépendre du contexte dans lequel il est utilisé. Parler du risque d'une installation nucléaire peut signifier l'accident qui est susceptible de s'y produire ou la probabilité de survenue de cet événement sur une période donnée ou dans des circonstances particulières. Dans d'autres cas, on associe au risque la mesure de la sévérité des conséquences d'un accident possible ("cet accident fait 10 morts") sans considération de sa probabilité. On peut aussi combiner probabilité et mesure de la sévérité, c'est la notion d'espérance mathématique qui conduit à considérer comme équivalents l'accident peu probable mais avec un grand nombre de victimes et l'accident fréquent avec peu de victimes.

Trois catégories d'acteurs sont impliqués dans la maîtrise des risques : les techniciens, les gestionnaires (économistes et administrateurs), et le public, qui ont chacun leur propre acception du mot risque et des modes d'évaluation et de gestion différents (Fig. 1).

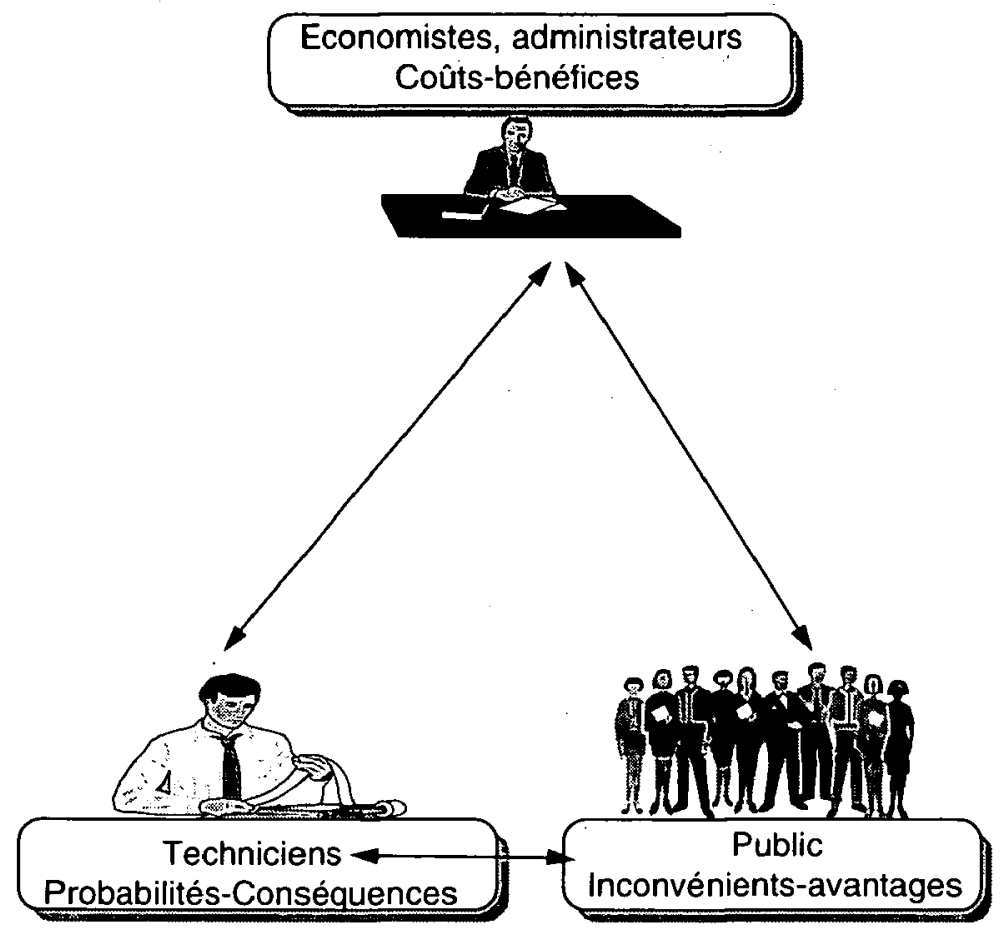

Fig. 1. - Trois visions du risque.

Three viewpoints on risk.

- Les techniciens ont une vision réductrice et opératoire du risque. Ils recueillent des statistiques sur la situation ou la modélisent; ils calculent des probabilités et les valeurs des conséquences, fournissent des prescriptions techniques et proposent des actions (choix d'options ou de procédures, normalisation). L'évaluation des expositions, des doses et des risques (en terme de mortalité ou de morbidité) auxquels sont exposés les populations et les travailleurs en situation normale ou accidentelle constitue l'essentiel de l'activité des techniciens de la radioprotection. 
- Les économistes et administrateurs ont une vision plus large. Ils traduisent le risque défini au plan technique par des coûts qu'ils confrontent aux bénéfices de la situation. L'élaboration de normes et la recherche. d'options optimisées pour prévenir et diminuer le risque sont de leur ressort. Pour la radioprotection, c'est là que se situent les choix des limites ou des niveaux d'intervention et les décisions consécutives à la mise en œuvre des procédures ALARA.

- Les individus du public perçoivent la situation de façon globale. La description qui en est faite mêle inconvénients et avantages qui souvent ne sont pas quantifiables. Aussi les individus s'appuient-ils sur tout un ensemble de critères qualitatifs pour juger la situation, pour traduire leur perception. Leur participation à la gestion du risque se fait selon des modes établis, ou en exerçant des pressions sur ceux qui gèrent. L'information et la communication sont deux volets de la participation, mais il existe bien d'autres modes institués par le législateur ou par les autorités politiques. Les situations radiologiques n'échappent pas à cette appréhension globale de la part du public tant du point de vue des risques que de la protection contre les rayonnements. Par contre pour les travailleurs impliqués dans les activités nucléaires, le contexte de travail intervient plus ou moins dans le jugement porté et les actions entreprises pour assurer leur protection.

\section{Modèles de perception du risque}

Les modèles proposés partent de l'hypothèse que la perception du risque résulte de la triple interaction entre :

- la situation et son risque "objectif”, c'est-à-dire un ensemble de conséquences jugées néfastes par un grand nombre d'individus et quantifiées dans le meilleur des cas, avec aussi leurs probabilités de survenue,

- l'individu face au risque, et

- l'environnement social dans lequel se situe le risque et où vit l'individu.

Le modèle SPR Stimulus - Personnalité - Réponse (Fig. 2) a été proposé par Slovic et al. (1980). Il s'agit d'un schéma mécaniste où la perception du risque se réduit à l'interaction entre la situation et l'individu. La situation à risque est qualifiée de réelle dans la mesure où ses fondements plus ou moins techniques sont considérés comme justifiés et indiscutables. Pour l'individu, la perception au premier niveau est celle des sens. Avec sa personnalité, son savoir et son expérience, l'individu construit une image, une représentation de la situation à risque. Viennent ensuite les attitudes (prédispositions à) qui se manifestent dans les opinions et comportements observés. Un problème se pose quand les opinions et comportements vis-à-vis du risque ne sont pas ceux que l'on attendrait d'après la description objective de celui-ci. On identifie alors la cause à une perception erronée. La solution du problème, pour ceux qui gèrent le risque, passe par l'augmentation du niveau de connaissance et la 
réduction de l'anxiété. Dans les activités quotidiennes, de tellẹs actions s'appliquent bien car les individus sont généralement intéressés par l'acquisition d'informations techniques et de règles de comportement sécuritaire améliorant leur protection. Les travailleurs du domaine nucléaire relèvent de ce dernier cas.

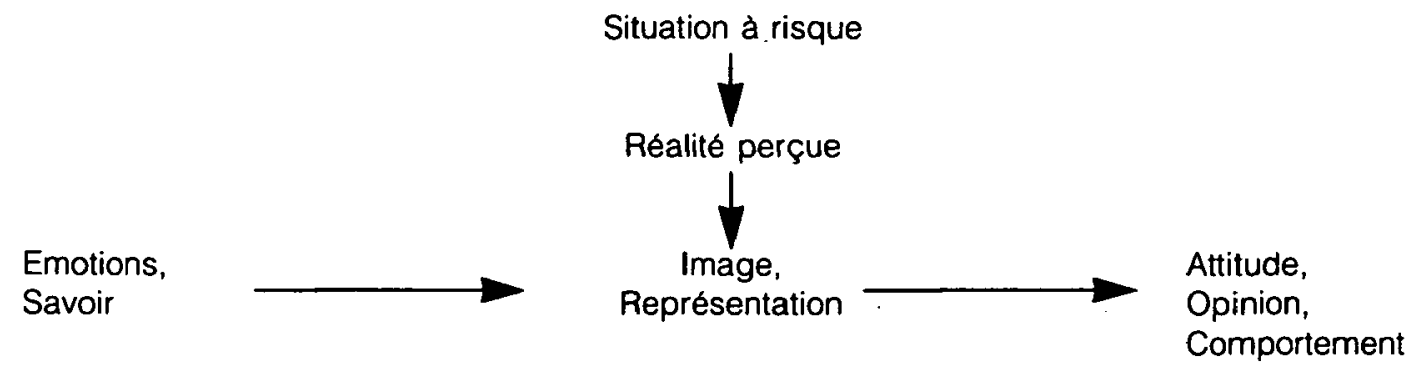

Fig. 2. - Perception des risques : schéma mécaniste.

Risk perception : a mechanistic scheme.

Le modèle SPR s'avère insuffisant pour la plupart des risques technologiques et situations dont l'individu n'a pas de vécu. Sa connaissance ne peut venir alors que des messages sur le risque émis par d'autres individus ou groupes et par les médias. Ces messages divers traduisent des points de vue particuliers; ajoutés aux réalités objectives de la situation, ils constituent la réalité transmise qui se révèle être souvent différente de la réalité objective telle que définie précédemment. Kasperson et al. (1988) utilise pour cela le terme d'“amplification sociale". S'agissant des médias, leur rôle est important dans tout débat sur le risque car ils disposent de souplesse et de liberté pour décrire la situation tant dans la forme que sur le fond. La sélection des risques par les médias, leur façon de les traiter ainsi que leur influence sur la perception (via l'amplification et la construction des représentations) ont été largement étudiées (Mazur, 1981). Mais l'individu, comme membre du public, n'est pas qu'un simple récepteur car il exprime un point de vue fondé sur les caractéristiques de la situation mais aussi sur son propre système de valeurs. Dans les cas extrêmes, généralement les situations conflictuelles et débattues au sein du public, l'individu sélectionne souvent parmi tous les arguments proposés ceux qui s'ajustent au mieux à son style de vie donc ceux qui sont en accord avec ses valeurs. Interviennent alors les arguments techniques ainsi que les habitudes culturelles et les normes sociales. Toutes les positions individuelles se fondent dans "l'opinion publique", qui se reflète dans les divers médias, les sondages et de façon ultime lors d'élections. L'opinion publique pèse à la fois sur ceux qui défendent et ceux qui s'opposent à la situation. Elle exerce toujours une influence sur leurs discours et leurs décisions, et leur donne parfois une légitimité. Cette pression du public sur les décideurs s'est particulièrement manifestée à propos des activités nucléaires. La figure 3 traduit cette dynamique de la perception des risques (Pages et al., 1988). 


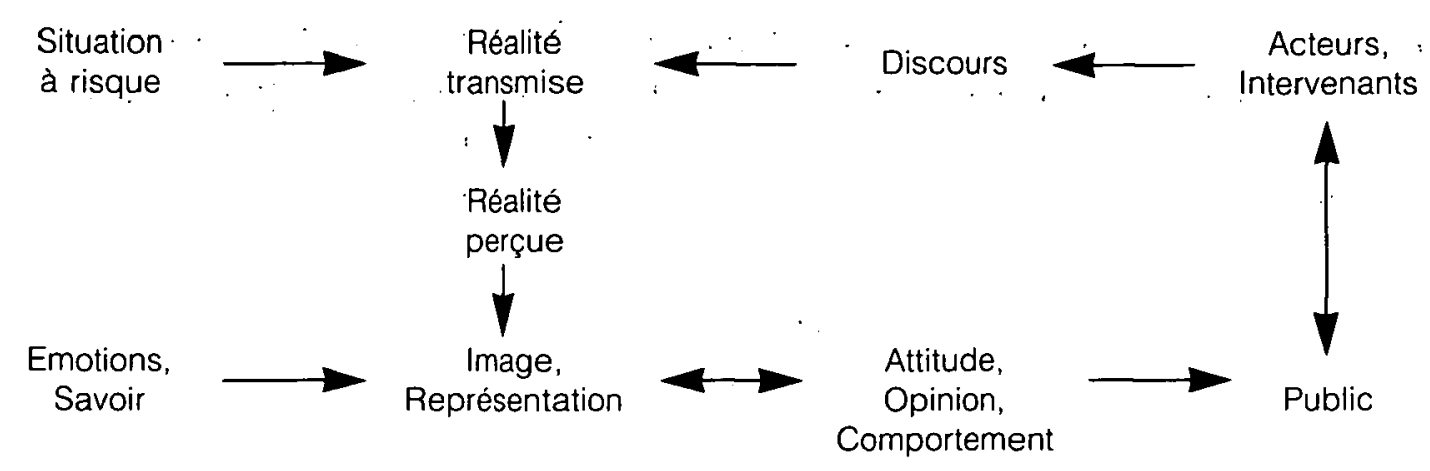

Fig. 3. - Perception des risques : schéma constructiviste. Risk perception : a constructivist scheme.

\section{Risques perçus et risques objectifs : une certaine cohérence}

La hiérarchie des situations en terme de risque perçu est relativement proche de celle, souvent jugée objective, basée sur des données statistiques ou des calculs. En figure 4, trente situations à risques sont repérées en abscisse par l'ordre des statistiques annuelles de décès et en ordonnée par l'ordre des nombres de morts par an estimés par le public (Bastide et al., 1989). Comme on

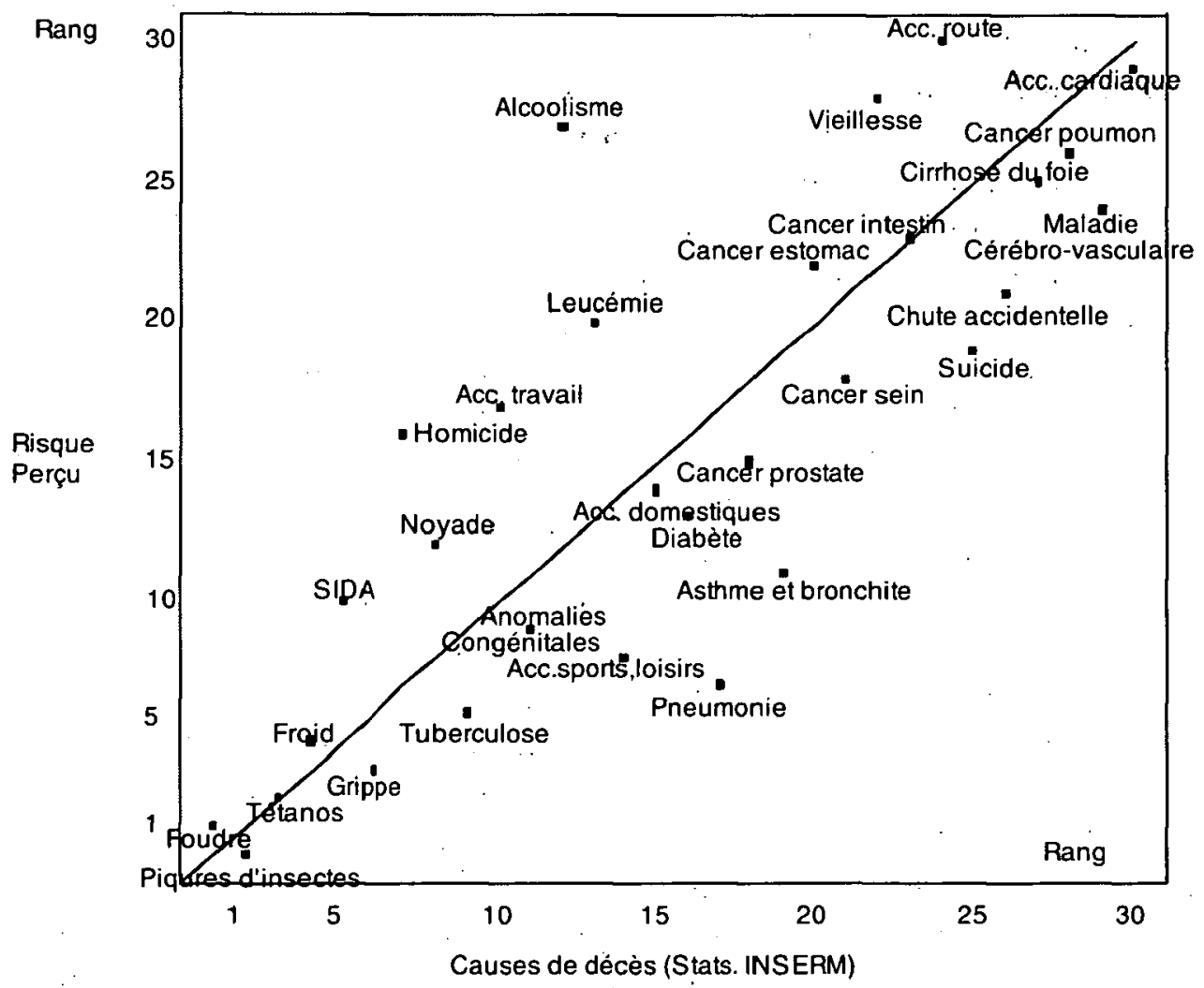

Fig. 4. - Classement des causes de mortalité.

Causes of death ranking (Source : enquête IPSN-IFOP, février 1986, 1000 personnes, France) 
peut le voir, l'ordre des causes de décès n'est pas sensiblement différent dans les deux cas. De faibles écarts apparaissent aussi dans la comparaison public/experts à propos du risque perçu. Dans une enquête réalisée à Bordeaux en 1989 (Barny et Brenot, 1991), les situations sont rangées de façon sensiblement analogue par les individus du public et les experts en sécurité, dont les avis sont considérés comme se rapprochant de l'objectivité, pour la question "dans les domaines ou situations d'accident cités, pensez-vous que les risques qui restent sont quasi-nuls, faibles, élevés ou très élevés?" (Fig. 5). Les écarts de perception les plus notables se manifestent pour les accidents domestiques et le transport des matières dangereuses qui préoccupent les experts, et la diminution de la couche d'ozone à laquelle le public se montre particulièrement réceptif.

Cette relative cohérence entre risques perçus et risques objectifs oriente vers la recherche de mécanismes plus sophistiqués que le simple déficit d'information pour expliquer les différences observées dans certains cas. La politique d'information ne peut être conduite en postulant que le public est ignorant voire incohérent.

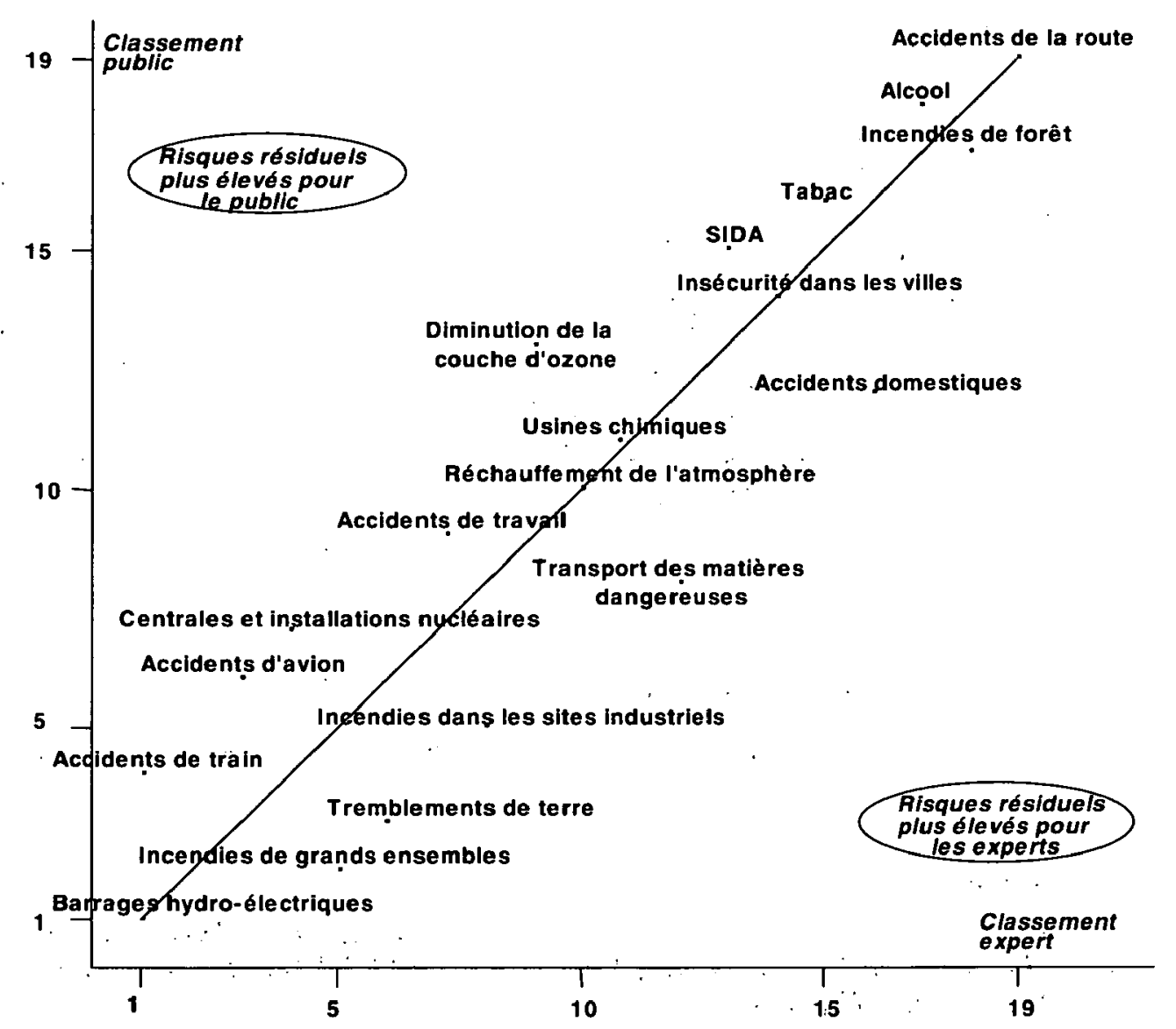

Fig. 5. - Classement sur le risque résiduel.

Residual risk ranking (Source : enquête IPSN-Bordeaux, octobre 1989, 150 experts, 706 bordelais) 


\section{Hiérarchie des risques perçus}

La hiérarchie des risques perçus est assez stable au cours du temps, mais aussi d'un pays à l'autre. Elle permet de relativiser certaines situations, comme par exemple les centrales nucléaires, et d'en identifier d'autres qui préoccupent durablement le grand public, comme les déchets nucléaires. Dans la figure 6 , apparaịssent les résultats de deux enquêtes faites simultanément dans le grand public aux Etats-Unis et en France en 1992 (Poumadere et al., 1994). En France, c'est pour les déchets radioactifs que le risque perçu des rayonnements est le plus élevé ; viennent ensuite à un degré moindre les centrales nucléaires, l'irradiation des produits alimentaires à but de conservation, et enfin à un niveau très bas les radiographies médicales et la radioactivité naturelle (le radon). On peut ainsi s'attendre à un public plus ou moins réceptif selon le thème traité. A ce titre, informer sur le radon qui est très mal connu et dont le risque est perçu si bas, représente une gageure.

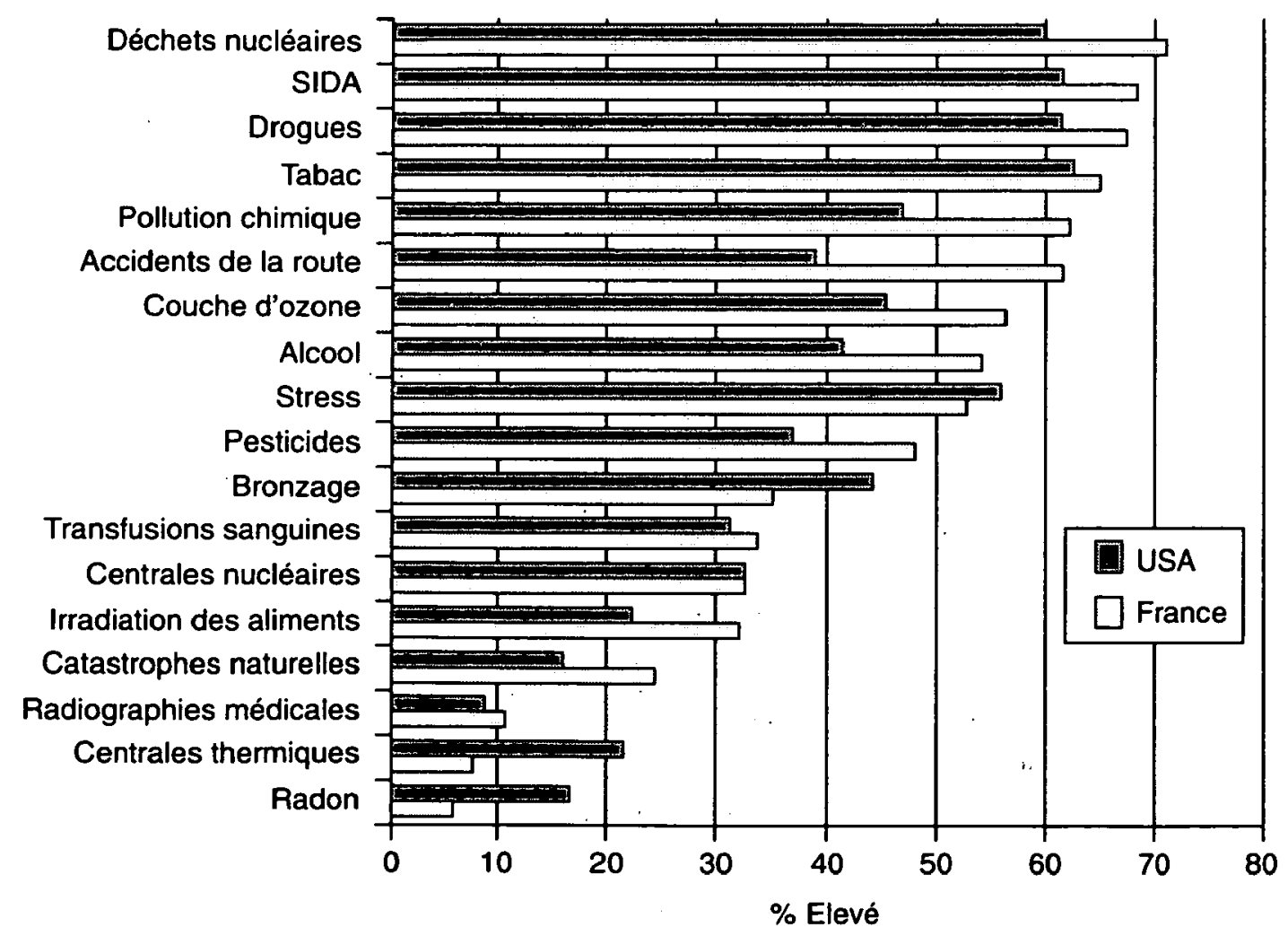

Fig. 6. - Hiérarchie des risques perçus.

Hierarchy of perceived risks (Source : enquête DECISION-RESEARCH, décembre 1992, 1550 personnes, USA ; enquête SYMLOG-BVA, décembre 1992, 1512 personnes, France) 


\section{Dimensions de la perception}

Dès les années 1970 dans la logique du modèle SPR, on a cherché à caractériser les situations à l'aide de tout un ensemble de dimensions qui expliqueraient le niveau de risque perçu. Ces dimensions sont nombreuses, jusqu'à 19 énumérées en figure 7 (Covello, 1985). Certaines dimensions concernent l'individu face au risque (de 1 à 7), d'autres la nature du risque (de 8 à 15), d'autres enfin la gestion sociale de celui-ci (de 16 à 19). Par exemple, on observe en général que le niveau de risque perçu diminue $(-)$ avec la familiarité de la situation, sa compréhension par l'individu, son contrôle et avec le fait que les bénéfices attendus sont tangibles; à l'inverse le niveau de risque perçu augmente $(+)$ quand la situation expose les générations futures ou les enfants, présente des conséquences qui ne semblent pas socialement équitables ou est telle qu'il existe un manque de confiance envers les institutions en charge de la gestion du risque.

Ces dimensions cependant ne sont pas toutes pertinentes pour chaque situation. Ainsi par exemple, l'immédiateté des conséquences qui généralement provoque un risque perçu plus élevé, ne s'applique pas aux déchets radioactifs, au radon ou aux radiographies médicales. On note même des effets à contrecourant, dans certains cas. Pour les activités nucléaires par exemple, on a souvent observé qu'une importante médiatisation peut améliorer leur compréhen-

\begin{tabular}{|clc|}
\hline 3. & Dimensions & Nivo \\
\hline 1. & La familiarité & - \\
2. & La compréhension & - \\
3. & L'incertain & + \\
4. & L'acceptation tacite & - \\
5. & L'implication personnelle & + \\
6. & Le contrôle personnel & - \\
7. & La valeur morale & - \\
8. & Le potentiel catastrophique & + \\
9. & L'existence d'un historique d'accidents & + \\
10. & Le caractère immédiat & + \\
11. & La réversibilité & - \\
12. & L'appréhension & + \\
13. & Les conséquences sur les enfants & + \\
14. & Les conséquences sur les générations futures & + \\
15. & L'identification des victimes & + \\
16. & L'équité & - \\
17. & Le bénéfice attendu & - \\
18. & La confiance envers les institutions & - \\
19. & La médiatisation & + \\
\hline
\end{tabular}

Fig. 7. - Dimensions de la perception.

Dimensions of perception. 
sion tout en augmentant le risque perçu. A l'évidence, les dimensions ne sont pas indépendantes ; ainsi par exemple, pour une activité à potentiel catastrophique élevé, l'appréhension est forte et le contrôle personnel inexistant. La complexité des liens entre ces dimensions et les perceptions montre que le modèle Stimulus-Personnalité-Réponse est insuffisant pour à lui seul expliquer . les perceptions et encore moins les attitudes vis-à-vis des risques, et qu'il ne peut être question de développer une information stéréotypée sur les risques. Pour aller plus loin, il est nécessaire de faire intervenir les aspects socio-politiques et socio-culturels.

\section{Variabilité des perceptions}

Pour une même situation, le risque perçu peut varier fortement d'un groupe d'individus à un autre. Ont été mis en évidence l'importance de facteurs psychologiques (comme l'anxiété), démographiques (tels que l'âge et le sexe), cognitifs (connaissance et expérience), sociaux (traduisant l'appartenance à un groupe comme le diplôme ou le revenu), et plus généralement culturels (modèles de société). La figure 8 illustre des différences significatives qui se manifestent au sein de divers groupes sociaux à propos de la perception des centrales nucléaires en France en 1993 (Brenot et Bonnefous, 1995).

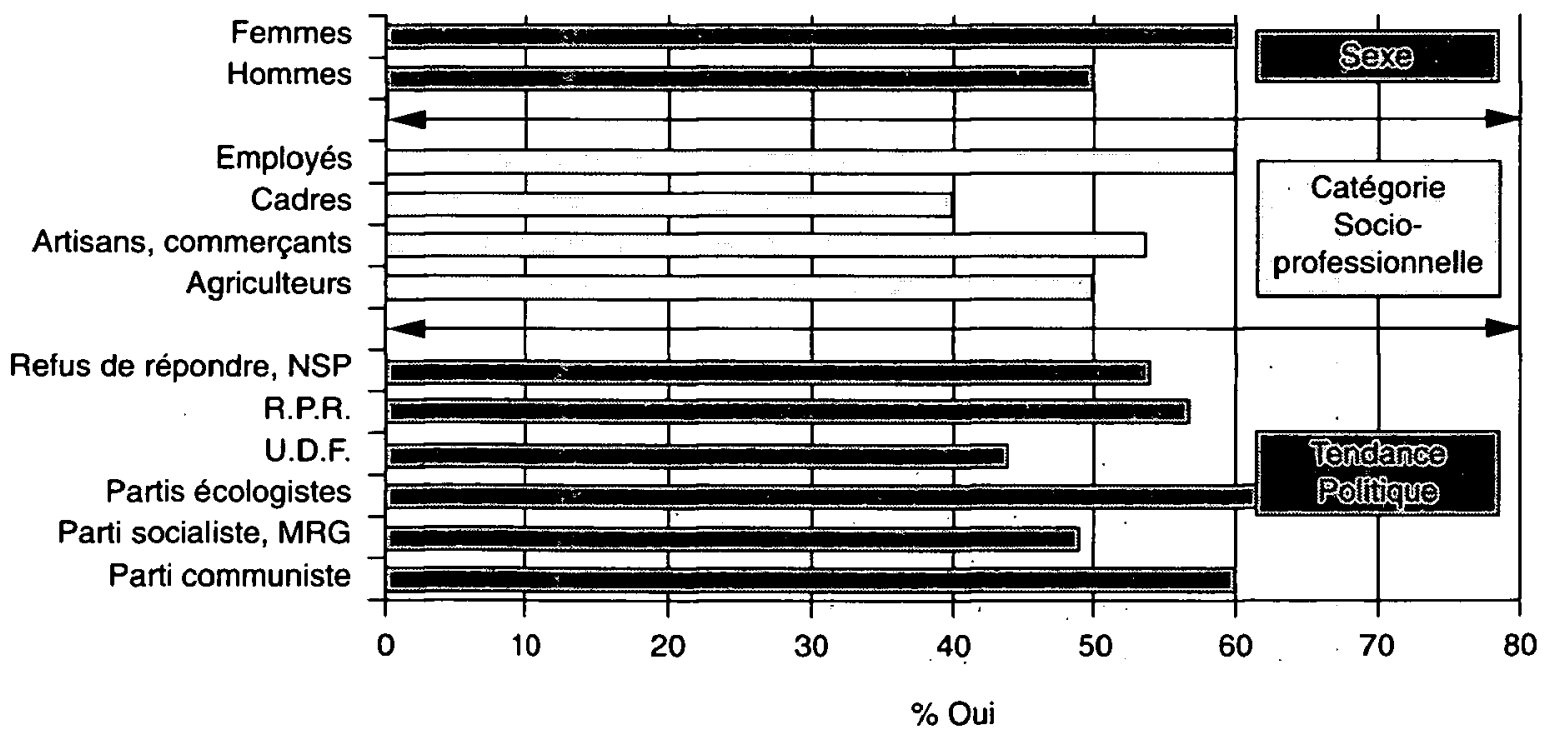

Fig. 8. - Perception du risque des centrales nucléaires.

Risk perception of nuclear power plants (Source : enquête IPSN-BVA, mai 1993, 1022 personnes, France)

\section{Espaces de perception}

Les situations peuvent être évaluées en terme de risque pour soi et ses proches ou de risque pour la société, ou encore selon l'urgence à prendre des 
mesures de sécurité. Quelle que soit la façon d'évaluer, les situations se-rangent en grandes catégories homogènes mises en évidence par des techniques statistiques. Ainsi, une analyse en composantes principales des réponses du public concernant vingt six situations évaluées sur l'échelle du risque pour soi et ses proches fait apparaître trois familles de situations dans la figure 9 : industrielles et technologiques sur la droite, personnelles au sud, et naturelles à gauche (Bonnefous et al., 1995). De façon générale, les activités industrielles ne sont pas jugées par le public de la même façon que les activités de loisirs, les activités domestiques, les modes de transport, les pratiques médicales ou les événements naturels. Aussi la comparaison de situations qui n'appartiennent pas à la même catégorie est souvent mal comprise du public. Par exemple, le radiodiagnostic et le risque radon ne peuvent être comparés aux installations nucléaires, et dans ces domaines les politiques d'information seront très différentes quant à la nature des contenus et moyens utilisés, des responsables et des cibles visés.

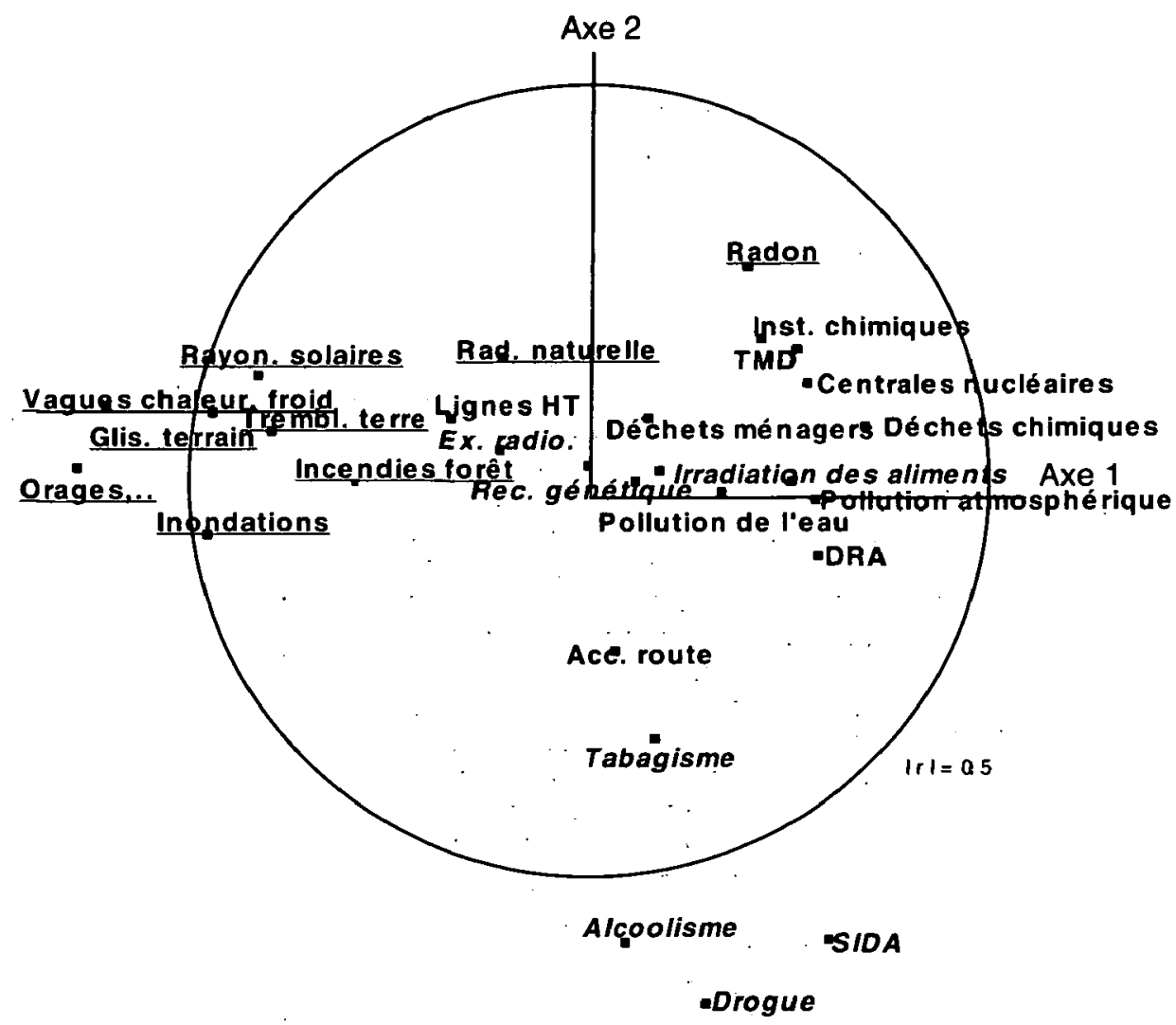

Risques: naturels; santé ; activités industrielles

Fig. 9. - Espaces de perception.

Map of perceived risks (Source : enquête IPSN-BVA, janvier 1995, 972 personnes, France) 


\section{Crédibilité de l'information}

Les individus du public manifestent dans tous les domaines une demande élevée d'information dès qu'il y a risque. Quant à la crédibilité de l'information diffusée, elle varie considérablement d'une situation à l'autre, comme le montre la figure 10 avec l'opposition entre les situations familières (accidents de la route, tabac, alcool, etc.) et les situations technico-industrielles (Bonnefous et al., 1994), opposition déjà observée par Lalo (1990). Pour les intervenants dans la gestion des risques, les travaux concernent surtout l'image de compétence et de crédibilité. Pour l'industrie et l'énergie nucléaires en France, les principaux intervenants institutionnels sont perçus comme compétents au plan technique alors que les associations de consommateurs, écologistes et médecins sont vus comme des sources d'information crédible. Ces images, fortement ancrées dans l'esprit du public, conditionnent en partie l'accueil réservé aux informations données et posent donc la question du choix du meilleur canal pour transmettre ces informations. Dans le cas des examens radiologiques, à qui faut-il destiner l'information sur les expositions? D'une part, l'individu du public n'en perçoit pas le risque et il s'en remet au corps médical pour ses expositions. D'autre part, les médecins ont une bonne image dans le domaine radiologique. Dès lors, on peut douter de l'intérêt d'informer prioritairement le public sur ce risque. Pour diminuer les expositions, l'effort d'information doit se porter d'abord vers les praticiens.

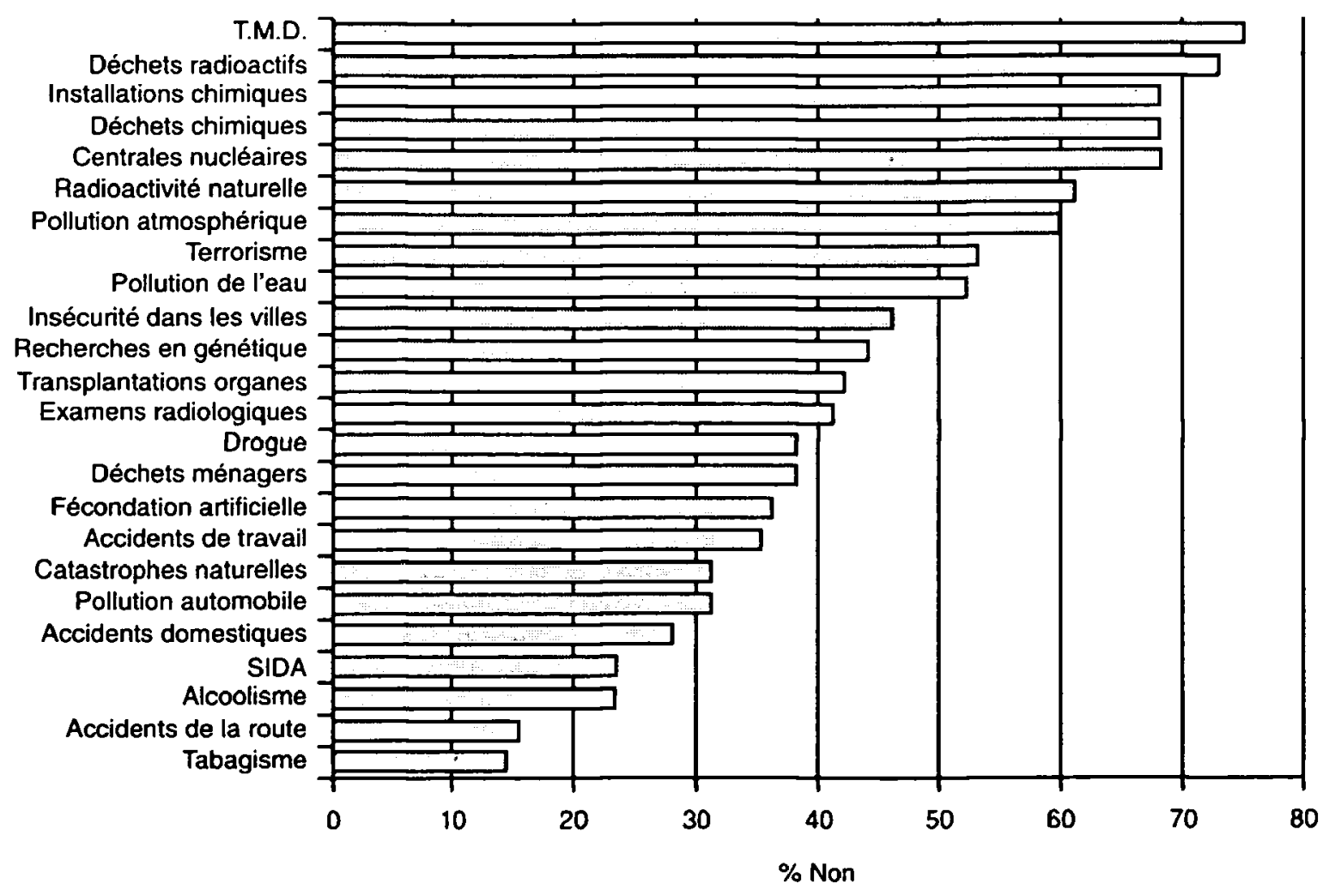

Fig. 10. - Véracité des informations sur les risques.

Truthfulness of the information on risks (Source : enquête IPSN-BVA, mai 1994, 942 personnes, France) 


\section{Conclusions}

La perception des risques dus aux rayonnements et son corollaire l'attitude vis-à-vis des activités nucléaires sont dans tous les pays reconnues comme étant d'une grande importance. Leur perception peut être analysée sur deux plans :

- au plan individuel : les individus inquiets et angoissés rejettent les activités nucléaires car ils refusent tout risque supplémentaire; il y a aussi ceux, qui ayant des connaissances techniques ou vivant à proximité d'une installation nucléaire expriment des jugements en général plus favorables aux activités nucléaires ; enfin, il y a tous les autres individus, c'est-à-dire la majorité, pour qui l'attitude la plus courante relative à une installation nucléaire est de dire "pas dans mon jardin", attitude à laquelle sont confrontés tous les projets d'installations industrielles importantes. Dans le cas des installations nucléaires, cette attitude s'explique par le fait que les risques apparaissent souvent mal connus et inquiétants, qu'il est impossible d'exercer un contrôle personnel, qu'il faut faire confiance, et que la situation n'est généralement pas équitable car les bénéfices sont nationalement répartis quand les risques sont localement subis.

- au plan sociétal : comme membres d'une communauté, les individus se sentent investis d'une responsabilité vis-à-vis des générations futures, et leurs perceptions des activités nucléaires sont fortement influencées par les acquis culturels, les positions politiques et idéologiques.

Les connaissances acquises sur la perception des risques nucléaires et la sécurité ont conduit les techniciens et les administrateurs à élargir leurs point de vue et à reconnaître leur troisième partenaire, le public. L'ouverture aux autres, l'écoute de discours sur le risque s'appuyant sur des logiques différentes (technique, économique, sociale) constituent une retombée immédiate des études sur la perception mais ce ne sont pas les seules. L'information sur les risques est guidée par les attentes et demandes qui se manifestent au cours des interviews. Pour le public, le pourquoi d'un risque est toujours secondaire par rapport au comment s'en protéger et les aspects techniques et économiques viennent loin derrière les aspects sanitaires ; aussi l'information sur la sécurité correspond à une forte demande. Certaines situations qui représentent des points de fixation doivent être abordées même si elles ne sont pas parmi les plus importantes d'un point de vue technique. Quant aux mesures de protection proposées par les autorités en situation normale ou accidentelle, serontelles acceptées et surtout appliquées? Incitation ou réglementation? Le port de la ceinture de sécurité ou la réduction du radon dans l'habitat sont deux exemples parmi bien d'autres, où la gestion du risque demande de savoir comment les actions de sécurité sont perçues par le public. Enfin la perception des acteurs de la sécurité, de leur compétence et de leur crédibilité, est un facteur essentiel de la confiance sans laquelle il n'y a pas de gestion de risque qui se puisse déléguer ni de réelle communication sur le risque. 


\section{Remerciements}

Ce travail a été réalisé grâce au soutien des Communautés Européennes dans le cadre du contrat CCE-DGXII-FI3P-CT930068.

\section{RÉFÉRENCES}

Barny M.H., Brenot J. (1991) La perception des situations à risques. Préventique 41, 11-19.

Bastide S., Moatti J.P., Pages J.P., Fagnani F. (1989) Risk perception and social acceptability of technologies : the French case. Risk Anal. 9 (2) 215-223.

Bonnefous S., Pommier S. Brenot J. (1994) Perceptions des risques et de la sécurité : résultats du sondage de mai 1994. IPSN, note SEGR/LSEES 94/42, juin 1994.

Bonnefous S., Pommier S., Brenot J. (1995) Perceptions dés risques et de la sécurité : résultats du sondage de janvier 1995. IPSN, note SEGR/LSEES 95/03, janvier 1995.

Brenot J., Bonnefous S. (1995) Approche socio-culturelle de la perception des risques. IPSN, note SEGR/LSEES 95/17, mai 1995.

Covello V.T. (1985) Environmental Impact Assessment, Technology Assessment, and Risk Analysis (V.T. Covello et al., Eds.) Nato Asi Ser. G, 4, 1-14, Springer Verlag : Berlin.

International Commission on Radiological Protection (ICRP) (1977) Recommendations ICRP Publication 26. Pergamon Press : Oxford.

Kasperson et al. (1988) The social amplification of risk : a conceptual framework. Risk Anal. 8 (2) $177-187$.

Lalo A. (1990) Informing the public on major technological risks : communication strategies of the Bouches-du-Rhône campaign April-June 1989. In : Communicating with the public about technological hazards (H.B.F. Gow and H. Otway, Eds.) Elsevier Appl. Sci. : London, pp. 204-231.

Mazur A. (1981) The dynamics of technical controversy. Washington D.C. Communication Press.

Pages J.P., Carde C., Brenot J., Bastide S. (1988) Analysis of risk perception. Decision context and approaches. In : Séminaire sur les applications, les perspectives et les limitations des évaluations comparatives des risques en vue de leur gestion, Nice, 26-30 septembre 1988. EUR Report 11465 EN, pp. 271-281.

Poumadere M., Mays C., Slovic P., Flynn J., Johnson S. (1994). What lies behind public acceptance? Comparison of US and French perceptions of the nuclear power option. In : Proceedings of the International Conference on the nuclear power option, IAEA, Vienne 5:8 sept. 1994, 393-405.

Slovic P., Fischhoff B., Lichtenstein S. (1980) Society Risk Assessment : How Safe is 'Safe Enough? (R: Schwing and W.A. Albers, Eds.), Plenum Press, New York, 181-214.

Travis C.C.; Hattemer-Frey H.A. (1988) Determining an acceptable level of risk. Environ. Sci. Technol: 22 (8) pp: 873-876. 